\title{
Polymerization Reactor Monitoring by In-line Raman
}

\section{Spectrometry}

Ramiro Infante Martínez, André Olivos Ramírez, Ricardo López González, Ramón Díaz de León, Luis Villarreal Cárdenas, Esther Treviño Martínez, Alejandro Díaz Elizondo and Beatriz Reyes Vielma

Procesos de polimerización, Centro de Investigación de Química Aplicada, Saltillo, Coahuila 25294, México

\begin{abstract}
This paper explores the feasibility of using in-line Raman spectrometry to monitor different monomer-solvent-polymer systems by different techniques: in-line, by an immersion probe; in-line, but externally aiming the spectrometer through the glass of the reactor wall; and off-line, taking the samples in small vials. The monitoring of chemical systems was done in the absence of polymer in order to simplify and facilitate the initial studies as well as in polymerization reactors operated with different processes: coordination polymerization of styrene-myrcene, anionic polymerization of myrcene, styrene emulsion polymerization, and methyl methacrylate - butyl acrylate mini-emulsion copolymerization. In all of these systems, it is of interest having an online monitoring technique for feedback control which would increase the productivity of the process, the quality of the product and the safety of the operation. Results show that Raman spectrometry was always capable to reproduce the trend of the process, with reasonable deviation with respect to gravimetric analysis.
\end{abstract}

Key words: Raman spectroscopy, polymerization reactor, in-line monitoring.

\section{Nomenclature}

$\begin{array}{ll}\text { 3D: } & \text { tridimensional } \\ \text { BA: } & \text { butyl acrylate } \\ \text { c: } & \text { concentration, gmol/L } \\ \text { BPO: } & \text { benzoyl peroxide } \\ \text { DEAC: } & \text { diethyl aluminum chloride } \\ \mathrm{I}_{0}: & \text { intensity of the irradiation beam, arbitrary units } \\ \mathrm{I}_{\mathrm{v}}: & \text { Raman signal intensity at of signal } \mathrm{v}, \text { arbitray units } \\ \mathrm{K}_{\mathrm{v}}: & \text { calibration constant } \\ \mathrm{MAO}: & \text { methylaluminoxane } \\ \mathrm{MMA}: & \text { methyl methacrylate } \\ \mathrm{NdV}_{3}: & \text { neodymium-versatate-catalyst } \\ \mathrm{PCA}: & \text { principal component analysis } \\ \mathrm{PLS}: & \text { partial least squares } \\ \mathrm{SDS}: & \text { sodium dodecyl sulfate } \\ \mathrm{SiO}_{2}: & \text { silicon dioxide } \\ \mathrm{Sty}: & \text { styrene } \\ \mathrm{V}: & \text { volume of the sample, } \mathrm{L}\end{array}$

\section{Introduction}

Due to the high demand, both in the industrial and in the academic environment, by inline measurement

Corresponding author: Dr. Ramiro Infante, research field: control of polymerization reactors. techniques for the monitoring and feedback control of polymerization reactors, there is a strong interest in the development of techniques susceptible of inline application such as calorimetry, densimetry, baro calorimetry, refractive index, optical spectrometry, etc. [1-3]. These techniques allow the real time monitoring of the reactors, as well as making possible the feedback control of important properties of the process, improving the quality of the product and increasing the productivity as well as the safety of the operation.

Inline spectrometry has been successfully applied in the monitoring and control of microstructure in rubber styrene-butadiene rubber [4]. The process study was the batch solution anionic polymerization of styrene and butadiene using an immersion probe optical fiber (near infrared). A phenomenological kinetic expression for the system that is in accordance with previously reported kinetic data was obtained. The authors considered further studies to develop strategies for the feedback control of the microstructure of the copolymer in semi-continuous 
and continuous operation.

Elizalde et al. [5] implemented inline calorimetry and Raman spectrometry for the monitoring of emulsion copolymerization of acrylic monomers and vinyl acetate. They found that both techniques produced comparable results of global conversion; regardless of the regime of operation (monomer starved or flooded conditions). However, the instantaneous conversions and the concentration of free monomer are determined more accurately by Raman spectrometry. Both techniques have the advantage of being non-invasive and are competitive in their application, with the potential for the development of techniques for monitoring and control of the process.

Van den Brink et al. [6] applied Raman spectroscopy inline to study the copolymerization in solution of styrene and butyl acrylate. The overlap of the vinylic peaks in this system did not allow direct application of univariate techniques, and so, multivariate approach was implemented, validating the results with gas chromatography off-line. Using the PCA (principal components analysis) technique, the main variation in the spectra was conversion of monomer, with a second largest effect attributable to variation in experimental/instrumental conditions. With the PLS (partial least squares) approach a univariate model was obtained for the concentration of the monomers. Both regression approaches gave satisfactory results for the calculation of the individual monomer conversions, showing concordance with off-line chromatography results.

Deshpande et al. [7] patented a method for tracking the polymerization of one or more olefin-type monomers in the presence of a solvent and at least one catalyst, with the polymer forming the dispersed phase, by Raman spectrometry. They monitored the concentration of the unsaturated carbon-carbon bond of the monomers by inline Raman spectrometry using an immersion probe of $785 \mathrm{~nm}$ (System RXN1 from Kaiser Optical Systems Inc.).
Fouconnier et al. [8] reported a technique of Raman spectroscopic analysis to monitor the Pickering emulsion polymerization of styrene with $\mathrm{SiO}_{2}$ support. These authors postulated that the ratio of signals at 1,630 and 1,000 wave numbers, corresponding to the vinyl group and the interaction of the silanol-aromatic ring, respectively, produced an estimate of the conversion with sufficient precision to dispense with a more complete chemical analysis such as that obtained by nuclear magnetic resonance.

The revision described in the papers mentioned above [2-8], shows that there is demand in the industrial and academic environment of spectroscopic techniques for inline monitoring and control of polymerization reactors. The present report considers the study of common monomers in order to test the spectroscopic technique and the diverse options of sampling modes (immersion probe, in vials, non-invasive probe) as well as to develop quantitative procedures for the spectra analysis (chemometrics).

\subsection{Beer-Lambert Law}

The Raman spectra quantitative treatment is based on the Beer-Lambert law, which relates the attenuation of light radiation of a definite wave number as it travels through a sample of certain chemical composition. This is an experimental law [9] common to optical spectroscopic technique. The Beer-Lambert law is expressed by

$$
\mathrm{I}_{\mathrm{v}}=\mathrm{cI}_{0} \mathrm{VK}_{\mathrm{V}}
$$

where, $I_{v}$ is Raman intensity of the radiation of wavelength $\mathrm{v}$ passing through the medium; $\mathrm{I}_{0}$ is the excitation Raman intensity; $\mathrm{V}$ is the volume of the sample illuminated by the source, $c$ is the concentration; $\mathrm{K}_{\mathrm{V}}$ is a constant characteristic for each band. Grouping the parameters that can be maintained constant and defining a global constant,

$$
\mathrm{K}=\mathrm{I}_{0} \mathrm{VK}_{\mathrm{V}}
$$

A linear expression for the concentration of the solute of interest in the medium is obtained:

$$
\mathrm{c}=\mathrm{I}_{\mathrm{v}} / \mathrm{K}
$$


A very clear demonstration of the use of Eq. (3) is the case of the emulsion homopolymerization in which the disappearance of monomer is easily detected by Raman spectroscopy of the reaction mixture and it is possible to quantify this concentration through the intensity of the signal of the vinyl group: $\mathrm{C}=\mathrm{C}$.

In the more general case of copolymerization or multi-polymerization, the situation is more involved, since monomers have signals of the double bond in each other at very close wavelengths. In that case the spectral contribution of additional functional groups characteristic of each monomer, as well as more rigorous mathematical techniques such as spectral deconvolution, and other linear methods such as minimum-squared based methods should be considered (principal component regression or partial least squares)[9] .

\section{Experimental Setup}

For ease of implementation and testing of the devices, the study was divided in: monitoring of monomer-solvent systems in the absence of reaction, and monitoring of various polymerization systems.

\subsection{Systems without Reaction}

Preliminary tests of different systems of monomer-solvent were studied by Raman spectrometry. This was done in order to explore, previous to the reaction monitoring step, the ability of Raman spectrometry as a method for in-line quantification. The systems studied were styrene-cyclohexane, myrcene-cyclohexane and myrcene-para methyl styrene-cyclohexane.

\subsection{Coordination Polymerization of Myrcene-Styrene in Solution}

A Zieggler-Natta catalytic system shown in Table 1 was used for the solution coordination polymerization of myrcene-styrene in cyclohexane.
Table 1 Catalytic system.

\begin{tabular}{lll}
\hline Reactants & Volume $(\mathrm{mL})$ & Concentration $(\mathrm{mol})$ \\
\hline MMAO & 6.9061 & 0.85 \\
$\mathrm{NdV}_{3}$ & 0.2996 & 0.56 \\
DEAC & 0.5032 & 1.00 \\
\hline
\end{tabular}

The polymerization was conducted in a jacketed stainless steel, batch reactor of 1 liter capacity. The temperature and pressure were maintained constant at $70{ }^{\circ} \mathrm{C}$ and $10 \mathrm{psig}$, respectively. A set of 10 tests was carried out in which the molar concentration of the monomers was varied, keeping constant the volume of the solvent $(280 \mathrm{~mL})$ and of the catalytic system.

\subsection{Anionic Polymerization of Myrcene}

Myrcene anionic polymerization was performed in a batch reactor using cyclohexane as solvent. Polymerization was monitored by high and low resolution Raman spectrometry (Smart Raman DXR2 and TruScan, respectively). Additionally, the record of temperature and operating pressure was done by means of a National Instruments hardware and software supervisory control data acquisition system (cDAQ and LabVIEW). Temperature control was performed using a temperature-controlled recirculating heating-cooling dynamic system (Presto, JULABO). The reaction time was 30 minutes in which it was possible to test the reactor by periodic sampling (cannula and tray) in vials of $2 \mathrm{~mL}$ for gravimetric analysis and Raman spectrometry.

\subsection{Emulsion Polymerization of Styrene}

The emulsion polymerization of styrene was performed using styrene, SDS (sodium dodecyl sulfate), as a surfactant, in deionized water as the dispersed phase, in amounts of 75:1.5:145 g, respectively. The initiators used were potassium persulfate and potassium metabisulphite.

The initiators were fed using an infusion pump of controlled flow. The reaction was started by the addition of $5 \mathrm{~g}$ of an aqueous solution with $30 \%$ of the total of the initiator; then, immediately the 
semi-continuous addition of $10 \mathrm{~g}$ of an aqueous solution with $70 \%$ of the remaining initiator began.

Raman spectrometry monitoring was made using samples in $2 \mathrm{~mL}$ vials and additionally focusing the laser beam of the portable system into the reaction medium through the window of the reactor wall.

\subsection{Mini Emulsion Polymerization of Acrylates}

The presence of emulsifier, a dispersing media, monomers, costabilizer and an initiator is characteristic elements in this type of polymerization. To form the mini emulsion, the mixture of monomers, initiator and costabilizer with the amounts shown in Table 2, undergo ultrasound for 15 minutes.

During polymerization, samples were taken for conversion calculations by gravimetry and for high and low resolution Raman spectrometry. Additionally, the portable Raman spectrometer was used focusing it into the reaction medium through the window of the reactor wall.

\section{Experimental Results}

\subsection{Dissolution of Styrene in Cyclohexane}

The incremental dissolution of 10 lots of styrene (10 mL each) in $150 \mathrm{~mL}$ of cyclohexane was monitored by means of a Raman probe. This allows analyzing the behavior of the mixture in the range of 0 to $62 \%$ of styrene, which covers the range of composition during a typical polymerization of this monomer.

The series of spectra obtained in the dissolution of styrene in cyclohexane is depicted in Fig. 1. It shows clearly how the characteristic signals of the insaturations of the styrene increase in intensity along the test.

The evolution of the concentration of styrene is obtained applying the Beer-Lambert law to signals in the region of wave number 1,560 to $1,700 \mathrm{~cm}^{-1}$. In Fig. 2 the real-time profile of the increase in the concentration of styrene is shown. Graphs such as this can be obtained in real time using the software
OMNIC of the spectrometer.

In Fig. 2 it can be seen that the online Raman probe achieved a good estimate of the concentration of styrene in the medium. As the data of the spectrometer were filtered (filter time of $3 \mathrm{~min}$ ), a slight delay in the estimated values can be observed.

\subsection{Dissolution of Myrcene in Cyclohexane}

The monitoring of the dissolution of myrcene in

Table 2 Miniemulsion recipe.

\begin{tabular}{ll}
\hline Component & Amount, g \\
\hline Hexadecane & 0.756 \\
BA & 9.45 \\
MMA & 9.45 \\
SDS & 0.756 \\
\hline
\end{tabular}

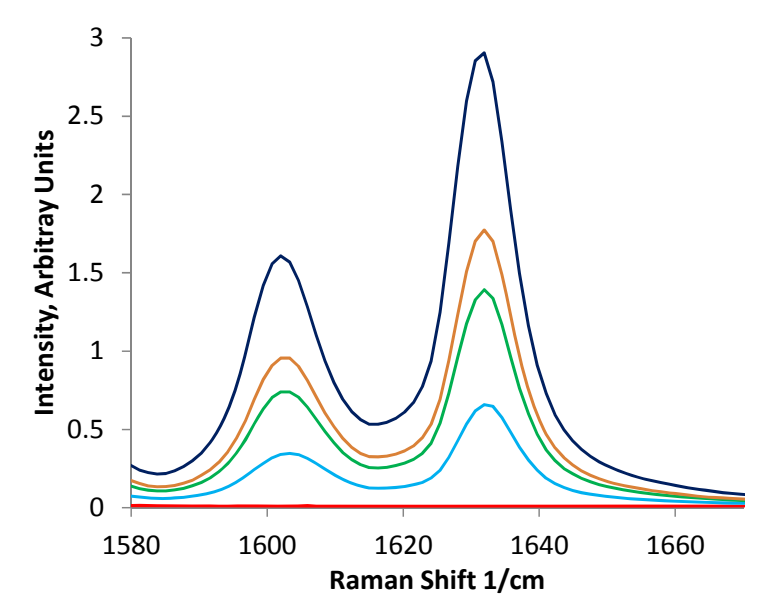

Fig. 1 Raman spectra in the dissolution of styrene in cyclohexane. Styrene concentration: $-0 \%,-19 \%,-38 \%$, $-50 \%,-62 \%$.

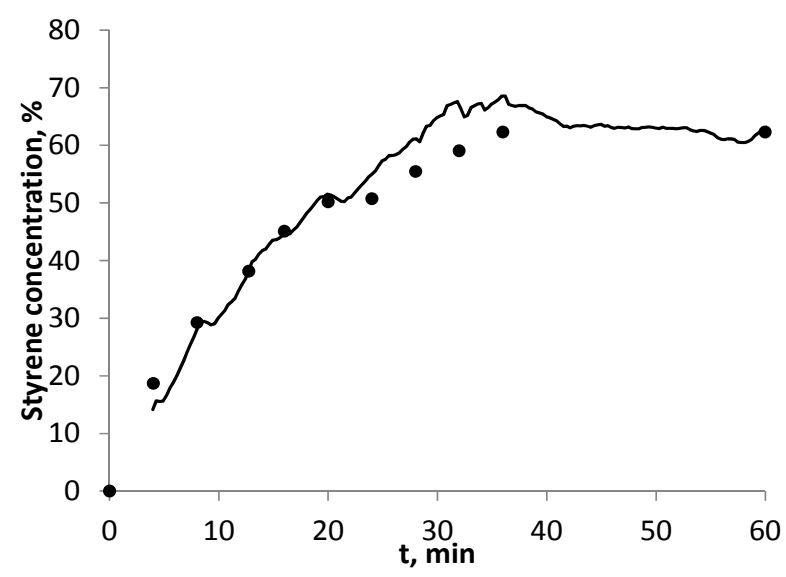

Fig. 2 Styrene concentration estimated by in-line Raman spectrometry (continuous line) and comparison with gravimetric analysis (dots). 
cyclohexane was done using a Raman probe in the dissolution medium (500 $\mathrm{mL}$ of cyclohexane). Myrcene was added continuously into an agitated vessel, the flow was controlled and constant at 2 $\mathrm{mL} / \mathrm{min}$ by means of a piston driven addition pump.

Fig. 3 shows the set of spectra obtained in real time during the dissolution of myrcene in cyclohexane. This is a $3 \mathrm{D}$ representation where it can be seen the characteristic signal of the $\mathrm{C}=\mathrm{C}$ bond present in myrcene, which appears around $1,635 \mathrm{~cm}^{-1}$. It can be seen the increasing intensity of the signal as the myrcene is added and dissolved in cyclohexane.

\subsection{Characterization of the Myrcene-Para Methyl} Styrene-Cyclohexane Ternary System

The high and low resolution Raman spectra of samples of known composition of the ternary system myrcene-para methyl styrene-cyclohexane were determined to cover the whole range of concentrations. This was done in order to study the sensitivity of Raman spectroscopy to quantify the composition of this system and then use this information for online monitoring of the copolymerization of this system.

Fig. 4 shows the series of spectra obtained for the binary case myrcene-cyclohexane. The increasing intensity of the monomer signal with the monomer concentration is clearly appreciated.

Fig. 5 shows the correlation obtained by applying the method of quantification of Beer-Lambert to this system.

It can be appreciated in Fig. 5 that although there is deviation in the estimation of the concentration of myrcene, this error might be acceptable to some feedback control techniques.

The most appropriate multivariate methods to analyze this ternary system are currently under consideration and will be reported in another communication.

\subsection{Myrcene-Styrene Solution Polymerization by Ziegler-Natta Coordination}

This system was monitored in the band of the spectrum of 1,630 to $1,640 \mathrm{~cm}^{-1}$ and the intensity of the signal with the solids content were correlated using the Beer-Lambert equation. Typical results obtained in

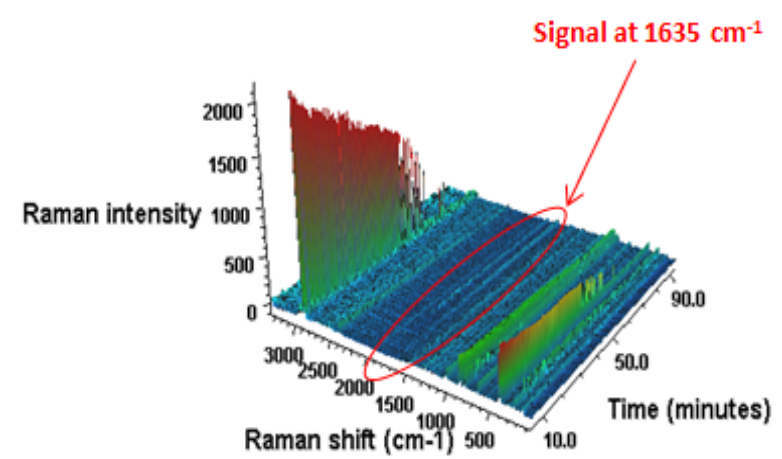

Fig. 3 The 3D representation of the increasing intensity of myrcene signal at $1,635 \mathrm{~cm}^{-1}$.

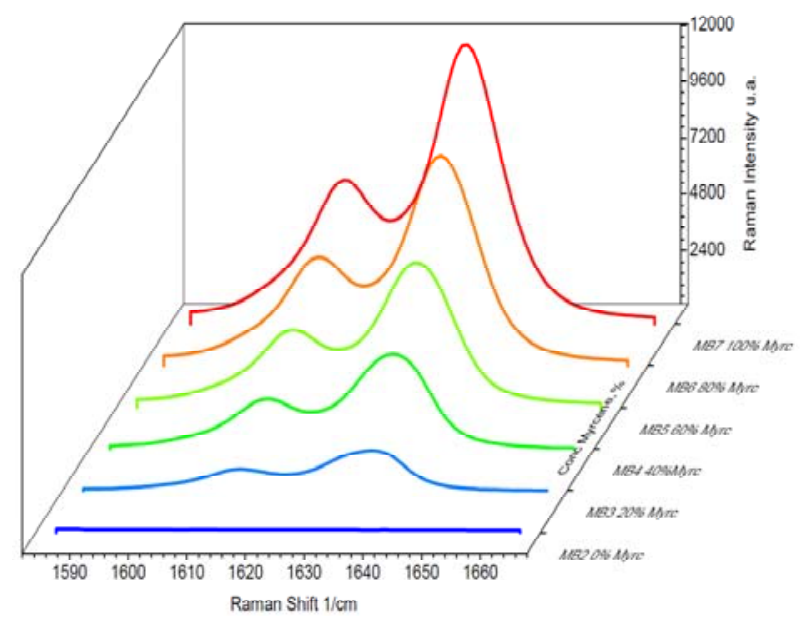

Fig. 4 Spectral evolution of the vinyl groups of myrcene monomer at increasing concentration. Concentration of Myrcene: $-0 \%,-20 \%,-40 \%,-60 \%,-80 \%,-100 \%$.

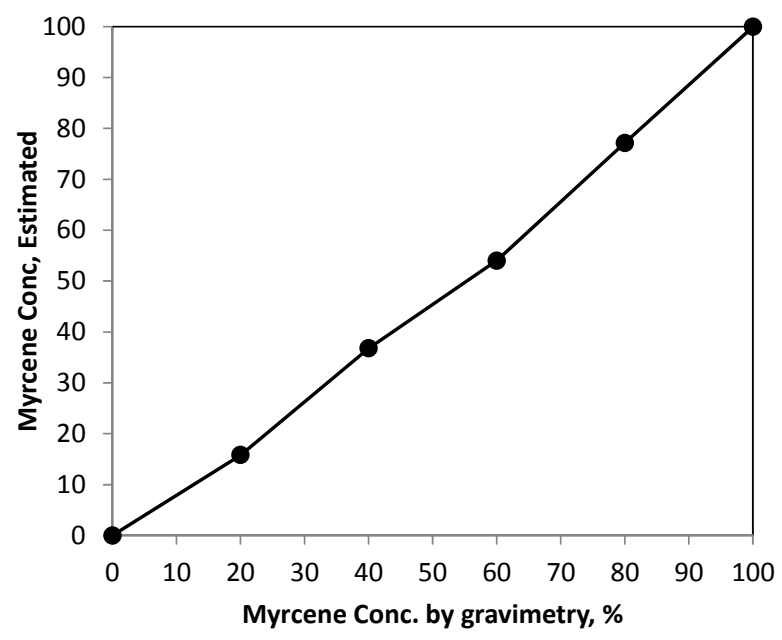

Fig. 5 Myrcene concentration estimated by Raman spectroscopy. 
the series of reactions are shown in Figs. 6 to 10. In this set of tests samples in vials were taken for Raman spectrometry and gravimetric analysis.

It can be noted the generally good correlation between quantification of solids content using Raman spectrometry and laboratory results. Greater deviation occurs at the start of the reaction, which is attributed that in the first few minutes occurs the greater instability of the reaction medium, and even heterogeneity of the system could occur. However, it is expected to have a better method of quantification when using the immersion probe for obtaining spectra, rather than sampling in vials.

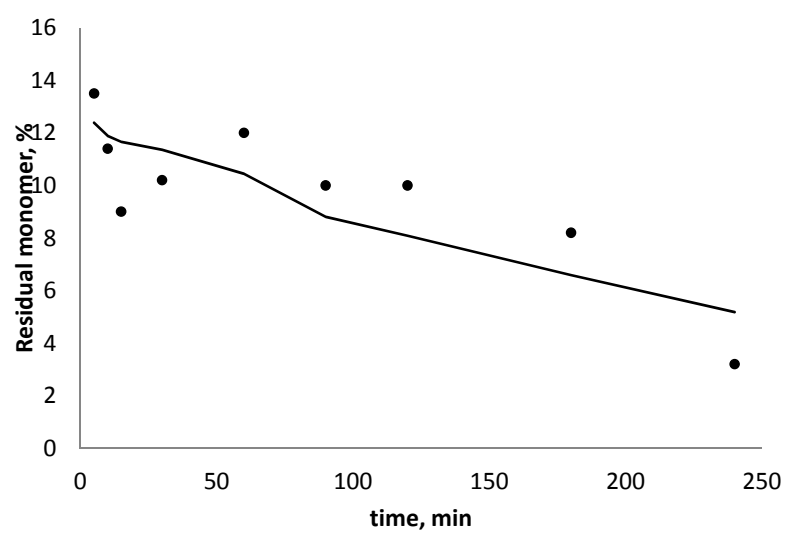

Fig. 6 Polymerization of myrcene-styrene (70\%-30\%). Raman estimation (dots) compared with gravimetric results (continuous line).

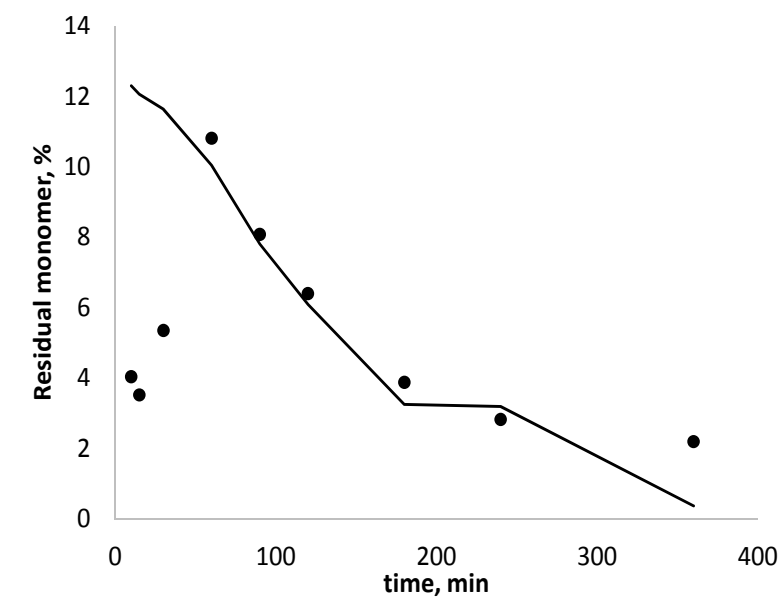

Fig. 7 Polymerization of myrcene-styrene (90\%-10\%). Raman estimation (dots) compared with gravimetric results (continuous line).

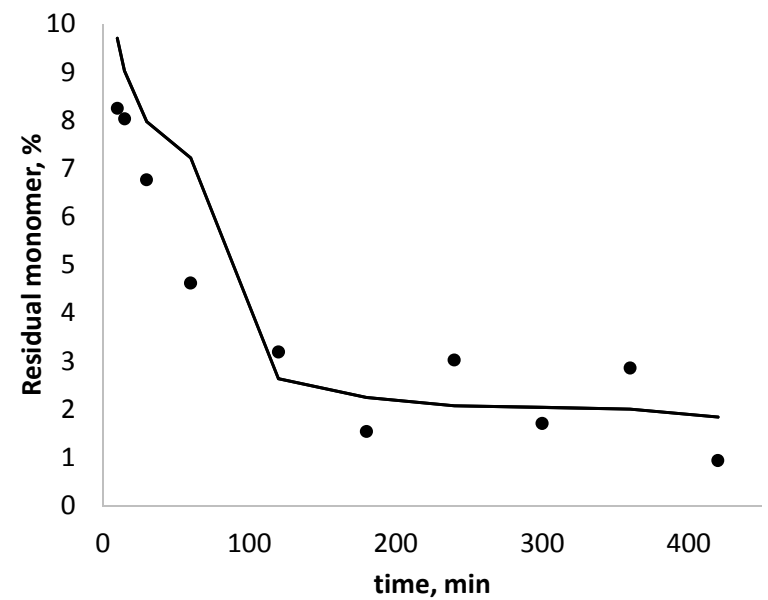

Fig. 8 Polymerization of myrcene-styrene (75\%-25\%). Raman estimation (dots) compared with gravimetric results (continuous line).

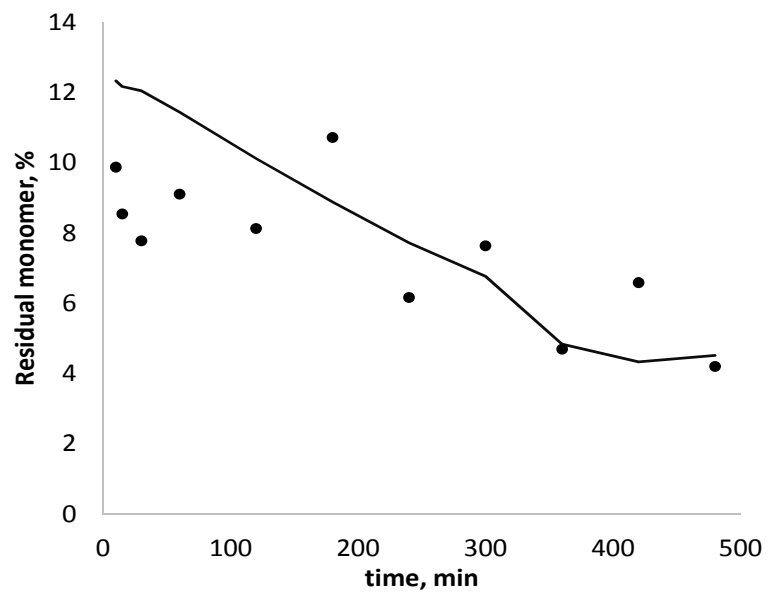

Fig. 9 Polymerization of myrcene-styrene (60\%-40\%). Raman estimation (dots) compared with gravimetric results (continuous line).

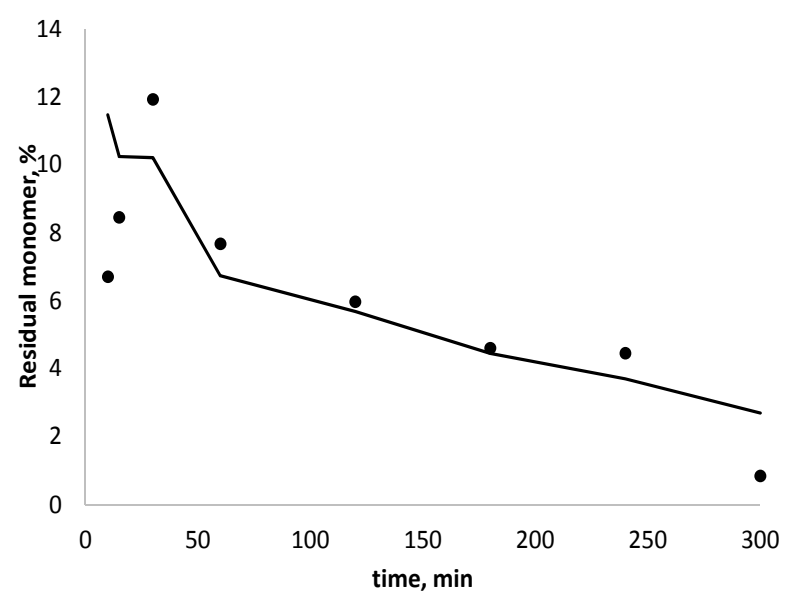

Fig. 10 Polymerization of myrcene-styrene $(80 \%-20 \%)$. Raman estimation (dots) compared with gravimetric results (continuous line). 


\subsection{Myrcene Anionic Polymerization}

As it was done in the previous polymerization systems, the reaction was followed by monitoring the disappearance of the signal of the monomer. Additionally, two signals in the regions of 500 to 550 $\mathrm{cm}^{-1}$ and $190-260 \mathrm{~cm}^{-1}$ were monitored, since these signals are present in the region of the aliphatic groups, and they can be correlated with the polymer formed during the reaction.

Fig. 11 shows that the estimates by Raman, via the Beer-Lambert law, applied to the vinyl group signal $\left(1,633 \mathrm{~cm}^{-1}\right.$ of Raman shift) correlates well with the solids content reported by gravimetry.

The estimation of the monomer content using the additional signals of 500-550 $\mathrm{cm}^{-1}$ and $190-260 \mathrm{~cm}^{-1}$ can be appreciated in Fig. 12.

It can be seen that the three signals considered for monitoring the reaction correlate well with the monomer content.

\subsection{Emulsion Polymerization of Styrene}

The monitoring of the reaction was conducted with high and low resolution Raman spectrometry taking samples at periodic intervals. The results in this system are not satisfactory, since they do not show the expected correlation between the vinyl group signal of the monomer and gravimetric results. This is attributed to the very complex system with certain heterogeneity and that specimens in vials do not ensure obtaining consistent and reliable spectra. Better results can be expected when the monitoring can be done by the immersion Raman probe technique in the future.

\subsection{Mini Emulsion Polymerization of MMA-BA}

In this test the monitoring of the polymerization of MMA-BA was carried out taking samples in small vials. The results with this method were not acceptable since the system presents heterogeneities that affect their Raman spectra and the results do not show consistency. On the other hand, the

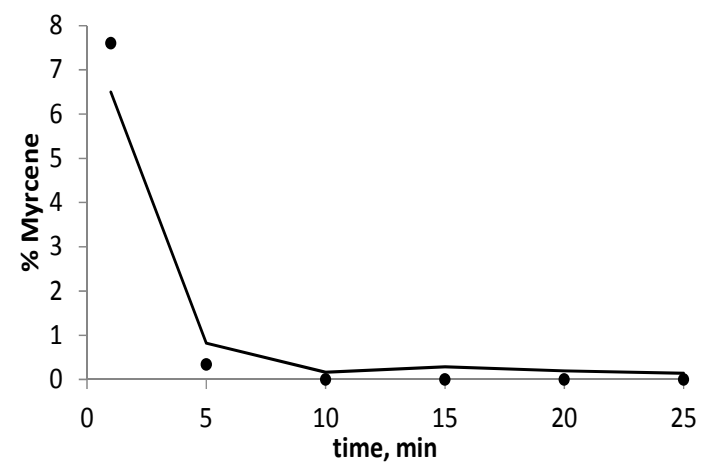

Fig. 11 Estimation of myrcene content by Raman spectrometry. Raman estimation (dots) compared with gravimetric results (continuous line).

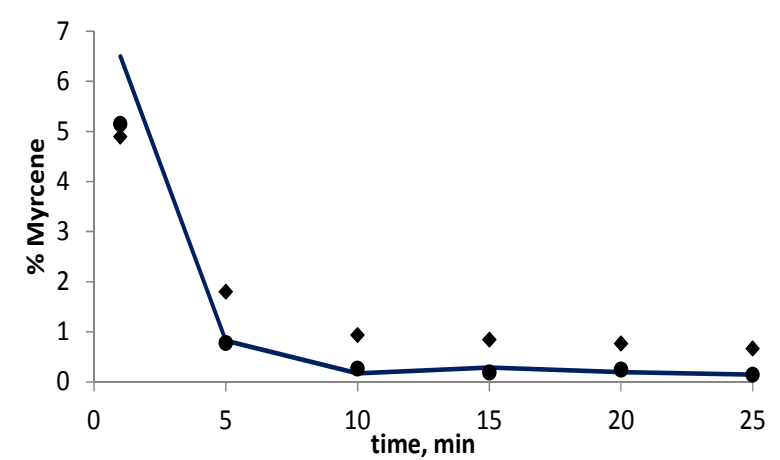

Fig. 12 Residual monomer estimates by two additional spectroscopic methods. Gravimetric results (continuous line), Raman estimates with signal at $500-550 \mathrm{~cm}^{-1}(\diamond)$, Raman estimates with signal at $190-260 \mathrm{~cm}^{-1}(\bullet)$.

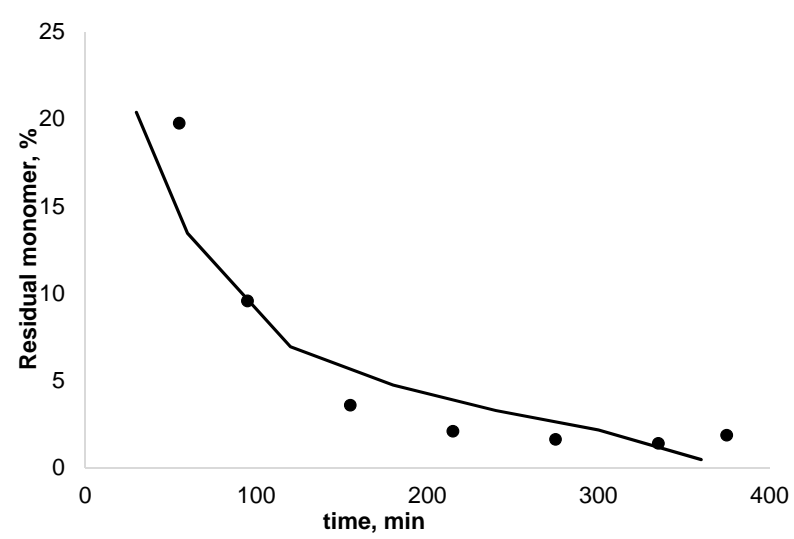

Fig. 13 Monitoring of the residual monomer in the MMA-BA mini-emulsion copolymerization. Gravimetric results (continuous line), Raman estimates (dots).

polymerization was monitored directly through the reactor using a portable Raman spectrometer obtaining better results with this technique. In Fig. 13 the quantification by Raman spectrometry and its comparison with gravimetric method is shown. 
Although there is error in the estimation, the results show that the use of an immersion probe to take the spectra data could improve the determination.

\section{Conclusions}

In general, good results were obtained for the monitoring of the various systems by Raman spectrometry. It is clear that the sampling technique (taking sample vials vs. immersion probe) plays a significant role in the precision of the determination in heterophasic systems such as emulsions and mini-emulsions. It is expected that in future studies these types of systems can be monitored via the immersion probe technique and obtain better results. However, it should be noted that even if it could occur error in the estimate, the trend (for example the disappearance of monomer), in all cases, is properly represented and in that sense, it can be said that the Raman spectrometry is one valuable tool with potential for the monitoring and control of the reactors in the systems studied here.

\section{Acknowledgments}

Financial support from CONACYT through grants 258278 (Basic Science Project); 271649 and 280485 (Laboratorio Nacional de Micro y Nanofluídica, Stablishment and Consolidation) is gratefully acknowledged.

\section{References}

[1] Wayne F. Reed, and Alina M. Alb. 2014. Monitoring
Polymerization Reactions from Fundamentals to Applications. ISBN 967-0-47-91738-1. Louisiana: John-Wiley.

[2] Infante, R., Cisneros A., Rivera C., and Jesús Alvarez. 2005. "A Baro-Calorimetric Technique for Monitoring Pressurized Copolymer Reactors." Journal of Applied Polymer Science 96: 475-82.

[3] Infante-Martínez, Jose R., Saldívar-Guerra Enrique, Pérez-Camacho Odilia, Comparán-Padilla, Víctor, and García-Zamora, Maricela. 2014. "Kinetic Monitoring Methods for the Ethylene Coordination Polymerization in a Laboratory Reactor." J. Appl. Polym. Sci. 131: 40035 .

[4] Rodríguez-Guadarrama, L. A. 2007. "Application of Online near Infrared Spectroscopy to Study the Kinetics of Anionic Polymerization of Butadiene." European Polymer Journal 43: 928-37.

[5] Oihana Elizalde, Maider Azpeitia, Marlon M. Reis, José M. Asua, and Jose R. Leiza. 2005. "Monitoring Emulsion Polymerization Reactors: Calorimetry versus Raman 10.1021/ie050451."

[6] Van den Brink, Jacques Franc, and Ois Hansen. 2001. "Measurement of Partial Conversions During the Solution Copolymerization of Styrene and Butyl Acrylate Using On-line Raman Spectroscopy.” Journal of Applied Polymer Science 79: 426-36.

[7] Deshpande, K., Stephenson, S., and Ravindra, D. 2013. Polymerization process and Raman analysis for olefin-based polymers. US Patent 2013/0261224

[8] Fouconnier, B., Terrazas-Rodriguez, J. E., and Lopez-Serrano, F. 2017. "Monitoring Styrene Pickering $\mathrm{SiO}_{2}$-Supported Emulsion Polymerization Kinetics by Raman Spectroscopy: Elucidating Mechanisms Interpreting the Silanol/Phenyl П-Interactions.” Journal of Macromolecular Science, Part A: Pure and Applied Chemistry 54 (8): 509-51.

[9] Workman, J. 1998. Applied Spectroscopy. A compact Reference for Practitioners. USA: Academic Press. 